\title{
Ethnic disparity in clinical performance between multivariate index assay and CA125 in detection of ovarian malignancy
}

\author{
Charles Dunton ${ }^{1}$, Rowan G Bullock*,1 \& Herbert Fritsche ${ }^{1}$ \\ ${ }^{1}$ Vermillion, Inc, Austin, TX 78738 \\ *Author for correspondence: rbullock@vermillion.com
}

\begin{abstract}
Aim: Based on evidence that African-American (AA) women have lower CA125 values than Caucasian (C) women, we investigated this to see if this disparity would have an impact on ovarian cancer detection using CA125 and multivariate index assay (MIA). Materials \& methods: Serum from two prospective trials of 1029 (274 malignancies [250 C/24AA]) were analyzed for CA125 and MIA results. Clinical performance was calculated. Results: Sensitivity of MIA in Caucasian women was $93.2 \%, 74.4 \%$ for CA125 at the ACOG approved cut-off level of $200 \mathrm{U} / \mathrm{ml}$ cutoff, and $80.4 \%$ using the 2007, Dearking $67 \mathrm{U} / \mathrm{ml}$ cutoff. In AA American women, MIA sensitivity was $79.2 \%, 33.3 \%$ for CA125 at the ACOG approved cut-off levels and $62.5 \%$ at the 2007 , Dearking $67 \mathrm{U} / \mathrm{ml}$ cutoff. Conclusion: Our results support that CA125 in AA women with adnexal masses has lower sensitivity than MIA no matter what the cutoff value is. Implementation of MIA in evaluation of adnexal masses should increase sensitivity of detection of malignancy compared with CA125, particularly in AA women.
\end{abstract}

First draft submitted: 29 May 2019; Accepted for publication: 8 July 2019; Published online: 9 August 2019

Keywords: biomarkers $\bullet$ CA125 • ethnic disparity • multivariate index assay • ovarian malignancy $\bullet$ race

A clinical challenge is identifying which women with an adnexal mass are at high risk of having a malignancy. Preoperative tumor marker testing, as well as ultrasound characteristics, have been utilized to differentiate between benign and malignant adnexal masses. Based on these characteristics, surgical referral to a gynecologic oncologist or treatment by a gynecologist can be undertaken. Recent publications have highlighted the differences in different ethnic populations of CA125 levels. Study populations have included healthy postmenopausal women, women with $B R C A$ mutations, women with invasive nonmucinous ovarian tumors, and women with ovarian cancer [1-4]. In each of these studies, lower values of CA125 were demonstrated in African-American (AA) women and/or non-White women. Based on this data, we identified a disparity in the detection of ovarian malignancies, as many adnexal mass assessment tools incorporate CA125, and use a universal cutoff regardless of the race of the patient. We investigated a database from two prospective studies, for an assessment of racial differences of the CA125 and the multivariate index assay (MIA), a serum test developed by Vermillion, Inc, which incorporates five biomarkers, intended for use in the clinical workup of suspicious adnexal masses [5,6]. We compared the sensitivity of CA125 with the MIA in both Caucasian women and AA women. Sensitivity, specificity, positive, and negative predictive values are presented for the entire cohort based on race.

\section{Materials \& methods}

Review of the database from the prospective studies conducted by Bristow in WIRB protocol number OVA2-001CO3 [5] and Ueland in WIRB protocol number OVA1-001-CO1 [6] of 1029 women which identified 274 women diagnosed with malignancy and for whom both CA125 and MIA were available. Cut-off values for CA125 were set at $>200 \mathrm{units} / \mathrm{ml}$ for premenopausal women and at $>35 \mathrm{units} / \mathrm{ml}$ for postmenopausal women, based on ACOG guidelines [7]. 
Table 1. Population demographics.

\begin{tabular}{|c|c|c|c|c|c|c|}
\hline \multirow[t]{2}{*}{ Demographic } & \multicolumn{3}{|c|}{ Caucasian subjects } & \multicolumn{3}{|c|}{ African-American subjects } \\
\hline & All malignancies & $\begin{array}{l}\text { Premenopausal } \\
\text { subjects }\end{array}$ & $\begin{array}{l}\text { Postmenopausal } \\
\text { subjects }\end{array}$ & All malignancies & $\begin{array}{l}\text { Premenopausal } \\
\text { subjects }\end{array}$ & $\begin{array}{l}\text { Postmenopausal } \\
\text { subjects }\end{array}$ \\
\hline \multicolumn{7}{|l|}{ Age (years) } \\
\hline $\mathrm{N}$ & 250 & 69 & 181 & 24 & 15 & 9 \\
\hline Mean (SD) & $58.0(12.9)$ & $44.1(8.4)$ & $63.3(10.0)$ & $48.5(13.1)$ & $41.5(9.1)$ & $60.2(10.1)$ \\
\hline Median & 57 & 47 & 61 & 48.5 & 42 & 62 \\
\hline Range (min, max) & 20,92 & 20,58 & 41,92 & 25,75 & 25,53 & 43,75 \\
\hline \multicolumn{7}{|l|}{ Pathology diagnosis, n (\%) } \\
\hline Primary ovarian malignancy & $167(66.8)$ & $43(62.3)$ & $124(68.5)$ & $12(50.0)$ & $9(60.0)$ & $3(33.3)$ \\
\hline $\begin{array}{l}\text { Low malignant potential } \\
\text { (borderline) }\end{array}$ & $47(18.8)$ & $16(23.2)$ & $31(17.1)$ & $5(20.8)$ & $2(13.3)$ & $3(33.3)$ \\
\hline $\begin{array}{l}\text { Nonprimary with metastatic } \\
\text { involvement of the ovaries }\end{array}$ & $22(8.8)$ & $7(10.1)$ & $15(8.3)$ & $5(20.8)$ & $3(20.0)$ & $2(22.2)$ \\
\hline $\begin{array}{l}\text { Nonprimary ovarian } \\
\text { malignancies with no } \\
\text { involvement of the ovaries }\end{array}$ & $14(5.6)^{\star}$ & $3(4.3)$ & $11(6.1)$ & $2(8.3)$ & $1(6.7)$ & $1(11.1)$ \\
\hline SD: Standard deviation & & & & & & \\
\hline
\end{tabular}

MIA incorporates CA125, transferrin, transthyretin (prealbumin), apolipoprotein A1, and $\beta$-2-microglobulin. The individual biomarker results are used to generate an ovarian malignancy risk score using a proprietary algorithm [6]. Numerical results range from 0.0 to 10.0, and risk of malignancy was stratified as follows:

- Premenopausal;

- Low probability of malignancy (OVA1 <5.0);

- High probability of malignancy (OVA1 $\geq 5.0)$;

- Postmenopausal;

- Low probability of malignancy (OVA1 <4.4);

- High probability of malignancy (OVA1 $\geq 4.4)$.

Inclusion criteria for the patients in this database have been described previously [5,6]. Briefly, samples were drawn from females greater than or equal to 18 years, with a documented pelvic mass planned for surgical intervention, and no history of malignancy in the previous 5 years [5,6]. Institutional review boards approved both studies where the data were obtained and all subjects gave informed consent $[5,6]$. Sensitivity, specificity, positive, and negative predictive values are provided for the entire cohort. The $95 \%$ CIs and p-values were calculated using a one-sided exact binomial option within SAS 9.4

\section{Results}

Serum samples from 274 women diagnosed with malignancies were analyzed for both CA125 levels and MIA. Of these, 250 women were Caucasian (C) and 24 AA.

Primary ovarian malignancy was diagnosed in 179 cases $(167 \mathrm{C} / 12 \mathrm{AA})$, metastatic disease to the ovary in an additional 27 cases (22C/5AA). Borderline or low malignant potential tumors accounted for 52 patients (47C/5AA). Non-primary tumors with no ovarian involvement were found in 16 women (14C/2AA).

Patient demographics are seen in Table 1.

MIA demonstrated a sensitivity for malignancy of $93.2 \%$ (89.3-95.9) for Caucasian women, and $79.2 \%$ (57.992.9) for AA women. CA125 demonstrated a sensitivity of 74.4\% (68.5-79.7) for Caucasian women and 33.3\% (15.6-55.3) in AA women, using the ACOG approved cut-off levels.

Due to the clinical state of Ovarian cancer with $>65 \%$ detected at late stage, MIA was intentionally designed for high sensitivity to not miss cancer and marginal specificity, thereby allowing some additional referrals. MIA demonstrated somewhat reduced specificity in Caucasian patients, 45.3\% (41.3-49.3), compared with CA125 at 87.6\%. A similar trend is seen in AA women, with a specificity value of $66.7 \%$ (57.9-74.6) for MIA, and CA125 of 93.9\%. Positive predictive value (PPV) is reduced in MIA compared with CA125. The PPV of MIA in Caucasian 


\begin{tabular}{|c|c|c|c|c|}
\hline Detection of malignancy & Sensitivity $95 \% \mathrm{Cl}$ & Specificity $95 \% \mathrm{Cl}$ & PPV $95 \% \mathrm{Cl}$ & NPV $95 \% \mathrm{Cl}$ \\
\hline MIA C & $93.2(89.3-95.9)$ & $45.3(41.3-49.3)$ & $40.8(36.7-44.8)$ & $94.2(91.6-96.9)$ \\
\hline CA125 C (ACOG cutoff) & $74.4(68.5-79.7)$ & $87.6(84.8-90.1)$ & $70.7(64.8-76.2)$ & $89.5(86.8-91.8)$ \\
\hline CA125 C (Dearking cutoff) & $80.4(74.9-85.1)$ & $79.1(75.7-82.3)$ & $60.7(55.2-66.0)$ & $91.0(88.2-93.2)$ \\
\hline CA125 C postmenopausal (>35 U/ml cutoff) & $82.3(76.0-87.6)$ & $79.3(74.3-83.7)$ & $70.3(63.6-76.3)$ & $88.3(83.9-91.8)$ \\
\hline MIA AA & $79.2(57.9-92.9)$ & $66.7(57.9-74.6)$ & $30.2(19.2-43.0)$ & $94.6(87.9-98.2)$ \\
\hline CA125 AA (ACOG cutoff) & $33.3(15.6-55.3)$ & $93.9(88.4-97.4)$ & $50.0(24.7-75.4)$ & $88.6(82.1-93.3)$ \\
\hline CA125 AA (Dearking cutoff) & $62.5(40.6-81.2)$ & $87.9(81.1-92.9)$ & $48.4(30.2-66.9)$ & $92.8(86.8-96.7)$ \\
\hline CA125 AA postmenopausal (>35 U/ml cutoff) & $66.7(29.9-92.5)$ & $87.2(72.6-95.7)$ & $54.6(23.4-83.3)$ & $91.9(78.1-98.3)$ \\
\hline
\end{tabular}

women is $40.8 \%$ (36.7-44.8) and in AA women it is 30.2\% (19.2-43.0). The PPV of CA125 was $60.770 .7 \%$ for Caucasian women, and $54.6 \%$ for AA women.

Negative predictive value (NPV) for MIA in Caucasian women was 94.2\%(91.6-96.9), compared with $91.0 \%$ depending for CA125. In AA women, the MIA NPV was 94.6\% (87.9-98.2), 92.8\% for AA women.

Table 2 details sensitivity, specificity, positive and negative predictive values, along with 95\% CIs. Table 2 also shows the performance parameters using cut-off values for CA125 at $>200$ units $/ \mathrm{ml}$ for premenopausal women and at $>35$ units $/ \mathrm{ml}$ for postmenopausal women, based on ACOG guidelines [7] and 67.0 units per $\mathrm{ml}$ for premenopausal women, as suggested by Dearking et al., 2007 [8].

\section{Discussion}

A number of publications have discussed the ethnic disparity in CA125 values in recent years. Pauler et al. [1] evaluated the CA125 levels of over 18,500 healthy postmenopausal women as part of a screening trial for ovarian cancer. Race was determined to be a predictor of baseline CA125, with average values being significantly lower in African women (median: 9.0; 95\% range: 4.0-26.0 units $/ \mathrm{ml}$ ) than in Caucasian women (median: 14.2; range: 6.0-41.0 units/ml; $\mathrm{p}<0.001)$.

Skates et al. [2] discussed a cohort of 3692 women with BRCA mutations. They found that premenopausal Asian women had a $24 \%$ lower CA125 value compared with women of other races, and postmenopausal black women had 22\% lower predicted CA125 values compared with white women.

Cramer et al. [3] performed preoperative treatment on 805 women who were found to have nonmucinous ovarian cancer. Non-White women were found to have a geometric mean value of CA125 43.8\% lower than that of white women, and women of Jewish ancestry had a higher CA125 value than other women in the study.

Babic et al. [4] reviewed 5091 women with invasive epithelial ovarian cancer who had CA125 measurements in 13 different studies participating in the ovarian Cancer Association Consortium. The median CA125 value in non-White women in this analysis was $35.2 \%$ lower than that of white women.

After observing the CA125 disparities in previous studies, we wanted to explore the impact this might have on the clinical performance of both CA125 and MIA with regards to detection of ovarian malignancy in women who had undergone imaging to confirm the presence of an adnexal mass. In any subset of patients, MIA displayed a higher sensitivity than CA125, with the greatest difference being in AA women. The detection of malignancy in AA women by CA125 alone is quite low.

Although MIA has a decreased sensitivity in AA women than in Caucasian women, this somewhat expected as CA125 is a component of MIA, but the performance of MIA is significantly better than that of CA125 alone, with a p-value of 0.003 using a one-sided exact binomial test.

A similar analysis was done for risk of ovarian malignancy algorithm (ROMA), and found that MIA detected more cases of malignancy than ROMA, which is comprised of CA125 and HE4, as well, though with a less marked difference [9].

As the number of AA patients in the studies which made up the analysis database for this research is fairly small, we presented data on all malignancies rather than dividing them based on histologic subtype. During our exploratory analysis, we performed these calculations, but due to the small sample sizes found the confidence intervals too wide to draw solid conclusions from. We are in the process of developing research opportunities to 
add further AA women with primary ovarian malignancies to our specimen repositories in order to confirm the results found in this analysis.

Clinicians should be aware of the limitations of CA125 testing in AA women with adnexal masses. The lower sensitivity of CA125 has a biologic basis in that non-Caucasian women have overall lower levels in general of CA125. Four separate papers have demonstrated this finding [1-4]. It is well known that PSA levels vary based on ethnicity.

At this time, MIA is more sensitive than CA125 in the entire population studied. However, this difference is most marked in AA women, for which CA125 has a very low sensitivity for prediction of ovarian malignancy. Adoption of MIA will improve referral of women with ovarian malignancy to the proper surgeon, particularly when it comes to AA women.

\section{Future perspective}

Within the next decade, this research could be a stepping stone toward closing the survivorship gap between Caucasian women and AA women, where ovarian cancer is concerned. Increased use of a more sensitive test such as MIA in minority women, and clinical awareness of the shortcomings of entrenched medical practices like CA125 could potentially increase early detection, which is key for improved survivorship.

\section{Summary points}

- A number of independent study cohorts have demonstrated that non-White women, and African-American (AA) women in particular, have lower CA125 values than Caucasian (C) women, all other variables being equal.

- We compared the performance of CA125 to multivariate index assay (MIA), both being adnexal mass risk assessment tools, with regards to their ability to detect ovarian malignancies in C and AA women.

- Samples came from two prospectively collected databases, and were analyzed retrospectively based on previously recorded CA125 values and MIA scores, and included a total of 274 cancers, of which 250 were C and 24 were AA.

- Primary ovarian malignancy was diagnosed in 179 cases (167 C/12 AA), metastatic disease to the ovary in an additional 27 cases (22 C/5 AA), borderline or low malignant potential tumors accounted for 52 patients (47 C/5 AA), and nonprimary tumors with no ovarian involvement were found in 16 women (14 C/2 AA).

- Multivariate index assay demonstrated a sensitivity for malignancy of 93.2\% (89.3-95.9) for Caucasian women, and $79.2 \%$ (57.9-92.9) for AA women, while CA125 gave a sensitivity of 80.4\% (74.9-85.1) in Caucasian women using lower cut off levels and 74.4 (68.5-79.7) using higher cutoff levels, but only $62.5 \%(40.6-81.2)$ and $33.3 \%$ (15.6-55.3) in AA women dependent on cutoff levels.

- Across the board, MIA demonstrated increased sensitivity over CA125, with the most marked difference being in premenopausal cancer detection.

- There is a relatively small number of AA cancers in this database, and we are exploring opportunities to expand the dataset and confirm these results.

- Implementation of MIA in AA adnexal mass cases could be clinically useful for patient management.

Data sharing statement

The authors certify that this manuscript reports the secondary analysis of clinical trial data that have been shared with them, and that the use of this shared data is in accordance with the terms (if any) agreed upon their receipt. The source of this data is: OVA2-001-CO3 OVA1-001-CO1. As the data is proprietary, no individual clinical trial data will be shared other than the statistical summaries already available.

\section{Authors' contributions}

C Dunton focused in design and conceptualization, drafting, data revision and final approval. RG Bullock carried out design and conceptualization, drafting, data analysis and final approval. H Fritsche performed design and conceptualization, critical editing, data revision and final approval.

Financial \& competing interests disclosure

This research and manuscript was funded by Vermillion, Inc. The authors have no other relevant affiliations or financial involvement with any organization or entity with a financial interest in or financial conflict with the subject matter or materials discussed in the manuscript apart from those disclosed.

Medical writing support was provided by Eric Harvey at Health Decisions, Inc. and was funded by Vermillion, Inc. 


\section{Ethical disclosure}

The authors state that all clinical trials referenced in this manuscript underwent Institutional Review board approval, and all human participants gave informed consent to have their data used.

\section{Open access}

This work is licensed under the Attribution-NonCommercial-NoDerivatives 4.0 Unported License. To view a copy of this license, visit http://creativecommons.org/licenses/by-nc-nd/4.0/

\section{References}

Papers of special note have been highlighted as: $\bullet$ of interest

1. Pauler D, Menon U, McIntosh M et al. Factors influencing serum CA125II levels in healthy postmenopausal women. Cancer Epidemiol. Biomarkers Prev. 10, 489-493 (2001).

- Found that race is a significant predictor of normal CA125 levels with average CA125II concentration in African (median: 9.0) to be lower than that in Caucasian women (median: 14.2; $\mathrm{p}<0.001$ ).

2. Skates S, Mai P, Horick $\mathrm{N}$ et al. Large prospective study of ovarian cancer screening in high-risk women: CA125 cut-point defined by menopausal status. Cancer Prev. Res. 4(9), 1401-1408 (2011).

- Found that postmenopausal black women had a 22\% reduction in predicted CA125 level among women with BRCA mutations.

3. Cramer D, Vitonis A, Welch W et al. Correlates of the pre-operative level of CA125 at presentation of ovarian cancer. Gynecol. Oncol. 119(3), 462-468 (2010).

- Found that among women with invasive nonmucinous tumors, the geometric mean value of CA125 in non-White women was 232, compared with 411 in Caucasian women.

4. Babic A, Cramer D, Kelemen L et al. Predictors of pretreatment CA125 at ovarian cancer diagnosis: a pooled analysis in the Ovarian Cancer Association analysis in the Ovarian Cancer Association Consortium. Cancer Causes Control 28(5), 459-468 (2017).

- Found that non-white race was associated with a $13 \%$ lower CA125 level than in white women with ovarian malignancy.

5. Bristow RE, Smith A, Zhang Z et al. Ovarian malignancy risk stratification of the adnexal mass using a multivariate index assay. Gynecol. Oncol. 128, 252-259 (2013).

- Validated multivariate index assay (MIA) on 494 subjects, finding a sensitivity of $95.7 \%$ overall, and an early-stage sensitivity of 91.4\%.

6. Ueland FR, DeSimone CP, Seamon LG et al. Effectiveness of a multivariate index assay in the preoperative assessment of ovarian tumors. Obstet. Gynecol. 117, 1289-1297 (2011).

- Compared the effectiveness of MIA with physician assessment and CA125, finding the sensitivities to be 93.0, 75.0 and 69-77.0\% depending on the cutoff respectively.

7. American College of Obstetricians and Gynecologists' Committee on Practice Bulletins - Gynecology. Practice Bulletin No. 174: Evaluation and Management of Adnexal Masses. Obstet.Gynecol. 128(5), e210-e226 (2016).

8. Dearking AC, Aletti GD, McGree ME et al. How relevant are ACOG and SGO guidelines for referral of adnexal mass? Obstet. Gynecol. 110(4), 841-848 (2007).

9. Dunton C, Bullock RG, Fritsche H. Multivariate index assay is superior to CA125 and HE4 testing in detection of ovarian malignancy in African-American women. Biomarkers Cancer 11 (2019).

- Compared the effectiveness of MIA to risk of ovarian malignancy algorithm, finding the AA sensitivity for MIA to be $79.1 \%$ and risk of ovarian malignancy algorithm to be $54.5 \%$. 
